\title{
Evaluation And Categorization Of Cryptography
}

\author{
Saragadam Sushma
}

Lecturer, Bhimavaram, A.P., India.

saragadamsushma203@gmail.com

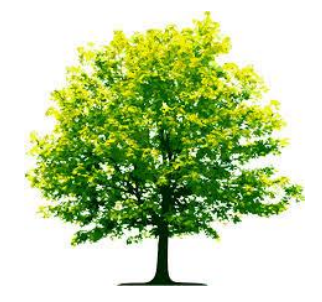

\begin{abstract}
A B S T RA C T
In the past cryptography was utilized as a part of keeping military data, conciliatory correspondence secure and in ensuring the national security. In any case, the utilization was restricted. These days, the scope of cryptography applications have been extended a considerable measure in the cutting edge zone after the advancement of correspondence implies cryptography is a method for defending the significant information from unapproved get to. It has risen as a protected means for transmission of data. It for the most part helps in checking interruption from outsider. It gives information privacy, respectability, electronic marks, and propelled client validation. The strategies for cryptography utilize science for securing the information (encryption and unscrambling).
\end{abstract}

Keywords: Cryptography,

Data Confidentiality,

Integrity, Electronic Signatures,

Authentication,

Encryption, Decryption
Citation: Saragadam Sushma (2018). Evaluation And Categorization Of Cryptography. International Journal of Advanced Multidisciplinary Scientific Research (IJAMSR ) ISSN:2581-4281 Vol 1, Issue 5, July, 2018, \#Art.517, pp 39-42

\section{Introduction}

Data security assumes a significant part amid web correspondence in the present time of innovation. It is colossally essential for individuals submitting eexchanges. For innocent individuals it might appear to be not that fundamental or expanded security may give solace to neurotic individuals, yet in all actuality it is significant when correspondence is conveyed between a huge number of individuals every day. There are different cryptography strategies that giveaway to secure trade and installment to private interchanges and ensuring passwords. Cryptography is essential for secure correspondences; it isn't independent from anyone else adequate. The per user of this paper will discover variations of cryptography and their applications. This paper has two noteworthy purposes. The first is to give 


\section{International Journal of}

Advanced Multidisciplinary Scientific Research (IJAMSR) ISSN:2581-4281

some genuine cases of cryptography being used today. The second is to give unthinkable synopsis and a conclusion.

\section{Cryptography}

Cryptography is the training and investigation of methods for secure correspondence within the sight of enemies. Regularly, it is tied with developing and examining conventions that conquer the impact of foes and which are identified with different angles in data security. Present day cryptography meets the controls of arithmetic, software engineering, and electrical designing. There are diverse sorts of cryptography. There is a sender, beneficiary, interloper of data and cryptographic device that keeps gatecrasher from trespass the touchy data.

\section{Types of Cryptography}

\section{Public Key Cryptography}

It includes two sets of keys: one for encryption and another for unscrambling. Key utilized for encryption is an open key and dispersed. Then again key utilized for decoding is a private key.

\section{Key Escrow Cryptography}

This innovation permits the utilization of solid encryption, yet additionally permits were acquired unscrambling keys held by escrow operators (outsider depended key escrow). The unscrambling keys are put into parts and given to isolate escrow specialists. Access to one a player in the key does not help decode the information; both keys must be gotten.

\section{Translucent Cryptography}

In this plan the administration can decode a portion of the messages, however not all. Just $p$ division of message can be unscrambled and 1-p can't be decoded. This is worthwhile over key escrow or no key escrow cryptography as whole data isn't at security hazard.

\section{Symmetric Key Cryptography}

Strategy utilizes same key for encoding and disentangling data. The sender and beneficiary of information must share the same key and keep data mystery keeping information access from outside.

\section{Use of Cryptography}

Cryptographic calculations are broadly being utilized to take care of issues having a place with information classification, information, trustworthiness, information mystery and validation and different spaces. It utilizes different cryptographic calculations as said above, according to the prerequisite of the activity. In the accompanying segment, the territories of the relevance of cryptography and its variations have been clarified. The measure of refinement among every one of the variations of cryptography is less on the grounds that the element in every one of the calculations is data that should be secured.

\section{Secure Message Transmission}

The intermediary signature plans enable intermediary endorsers to sign messages for the benefit of a unique underwriter, an organization or an association. It depends on the discrete logarithm issue. The sign encryption is an open key crude that at the same time plays out the elements of both computerized mark and 


\section{International Journal of} Advanced Multidisciplinary Scientific Research (IJAMSR) ISSN:2581-4281

encryption. Reconciliation of intermediary signature and sign encryption open key standards gives secure transmission.It is effective as far as calculation and correspondence costs. It is utilized for low power PCs in which a given gadget may transmit and get messages from a discretionarily huge number of different PCs.

\section{Communication Monitoring}

Cryptography can give colossally vigorous encryption; it can hinder the administration's endeavors to truly perform electronic observation. With a specific end goal to address this issue, the key is escrowed by means of endowed outsider.This innovation permits the utilization of solid encryption, yet in addition permits the administration when legitimately approved to get unscrambling keys held by escrow specialists.

\section{Fractional Observation of Data}

Here and there sender needs just piece of the message to be observed however not all. All things considered Translucent cryptography is utilized that investigates the space between misty (solid encryption with no key escrow) and straightforward (no encryption or encryption with key escrow). With translucent plan, the administration can decode a portion of the messages, yet not all. Similarly, as a translucent entryway on a shower slow down gives some protection, however, not immaculate security, translucent cryptography gives a few correspondences securities, yet not flawless security.

\section{Transferring Files on Network}

Documents that are to be traded between clients should be insured against pernicious clients and assailants. Symmetric Key cryptographic uses just single key for both encryption and unscrambling. In this innovate symmetric key is then scrambled with open key which is related with the sender of the document to get encoded record and this scrambled document is then sent to the recipient. To decode the document, encoded record framework segment driver utilizes private key which is related with the recipient to unscramble the symmetric key used to scramble record. The scrambled document framework segment driver is then used symmetric key to unscramble the record.

\section{Certificates and Authentication}

An authentication is an electronic record which distinguishes an individual, a server, an organization, or some other substance and to connect that character with an open key. Declaration specialists (CAs) issued authentication, which ties a specific open key to the name of the substance that the testament distinguishes (the name of a worker or a server). Notwithstanding, it, a declaration incorporates a serial number, name of endorsement specialist who issued it. And furthermore, it incorporates advanced marks of the issuing CA. Endorsements help keep the utilization of phony open keys for pantomime. Just the general population key ensured by the testament will work with the relating private key controlled by the substance distinguished by the authentication.

\section{Quantum Key Distribution}

It is the best known use of quantum cryptography. It is a procedure to build up quantum correspondence between the two gatherings for sharing a key (typically Alice and Bob), the outsider don't know anything about the key. This can be accomplished when Alice encodes the bits previously sending it to the Bob. 


\section{Conclusion}

In this exploration paper the pertinence of cryptography in information security has been contemplated and condensed. Likewise the different cryptographic strategies have been watched and their particular zones of naturalness have been discovered and an abridged table has been created.

\section{References}

[1]. A Survey on the Applications of Cryptography. Shivangi Goyal. 2012, International Journal of Scienceand Technology Volume 1 No. 3, 137-140.

[2]. Advance cryptography algorithm for improving data security. Vishwa gupta, Gajendra Singh, Ravindra Gupta. 2012, International Journal of Advanced Research in Computer Science and Software Engineering.

[3]. An overview of modern cryptography. Ahmed AlVahed and Haddad Sahhavi.2011, World Applied Programming, Vol (1), No (1), 55-61.

[4]. Data Hiding in Image using least significant bit with cryptography. Mr. Vikas Tyagi. 2012 , International Journal of Advanced Research in Computer Science and Software Engineering, pp. 120-123.

[5]. Survey Paper: Cryptography Is The Science Of Information Security. Mohammed AbuTaha, Mousa Farajallah, Radwan Tahboub, and Mohammad Odeh.2011, International Journal of Computer Science and Security (IJCSS), Volume (5) : Issue (3) 